\title{
On the development of polarizable and Lennard-Jones force fields to study hydration structure and dynamics of actinide(III) ions based on effective ionic radii
}

Cite as: J. Chem. Phys. 147, 161707 (2017); https://doi.org/10.1063/1.4989969

Submitted: 26 April 2017 . Accepted: 13 June 2017 . Published Online: 05 July 2017

Riccardo Spezia (D), Valentina Migliorati (D), and Paola D'Angelo (D)

\section{ARTICLES YOU MAY BE INTERESTED IN}

A general intermolecular force field based on tight-binding quantum chemical calculations The Journal of Chemical Physics 147, 161708 (2017); https://doi.org/10.1063/1.4991798

Mapping the Drude polarizable force field onto a multipole and induced dipole model The Journal of Chemical Physics 147, 161702 (2017); https://doi.org/10.1063/1.4984113

Toward chemical accuracy in the description of ion-water interactions through many-body representations. Alkali-water dimer potential energy surfaces

The Journal of Chemical Physics 147, 161715 (2017); https://doi.org/10.1063/1.4993213

The Journal

Submit Today

of Chemical Physics

The Emerging Investigators Special Collection and Awards Recognizing the excellent work of early career researchers! 


\title{
On the development of polarizable and Lennard-Jones force fields to study hydration structure and dynamics of actinide(III) ions based on effective ionic radii
}

\author{
Riccardo Spezia, ${ }^{1, a)}$ Valentina Migliorati, ${ }^{2}$ and Paola D'Angelo ${ }^{2}$ \\ ${ }^{1}$ LAMBE, Université d'Evry Val d'Essonne, CEA, CNRS, Université Paris Saclay, F-91025 Evry, France \\ ${ }^{2}$ Dipartimento di Chimica, Università di Roma "La Sapienza," Roma, Italy
}

(Received 26 April 2017; accepted 13 June 2017; published online 5 July 2017)

\begin{abstract}
In this contribution, we show how it is possible to develop polarizable and non-polarizable force fields to study hydration properties of a whole chemical series based on atomic properties such as ionic radii. In particular, we have addressed the actinide(III) ion series, from $\mathrm{U}^{3+}$ to $\mathrm{Cf}^{3+}$, for which $\mathrm{X}$-ray absorption data and effective ionic radii are available. A polarizable force field has been reparameterized improving the original one [M. Duvail et al., J. Chem. Phys. 135, 044503 (2011)] which was based on solid state ionic radii. The new force field does not depend on solid state properties but directly on the liquid phase ones, and it can be used to study these ions in liquid water without any ambiguity. Furthermore, we have shown that it is possible to parameterize also a non-polarizable potential using standard Lennard-Jones and Coulombic forces, which can be transferred to other systems in condensed phase. The structural and dynamical properties of these two force fields are compared to each other and with data available in the literature, providing a good agreement. Moreover, we show the comparison with experimental X-ray absorption data that are very well reproduced by both force fields. Published by AIP Publishing. [http://dx.doi.org/10.1063/1.4989969]
\end{abstract}

\section{INTRODUCTION}

Actinides are very relevant products of nuclear industry, and understanding their properties in liquid water is fundamental to rationalize their behavior in different situations: from stocking sites to reprocessing factories up to their behavior in the natural environment where they can migrate due to contamination. Heavy actinides (from Am) are present only as 3+ cations, while lighter ones can have different oxidation states, also forming oxocations (like in the case of uranyl, $\mathrm{UO}_{2}^{2+}$, which is the most abundant form in which this element can be found). ${ }^{1}$ Note that heavy actinides can be stabilized as $3+$ cations at very low $\mathrm{pH}$ conditions. The case of $3+$ actinides, $\mathrm{An}^{3+}$, provides an interesting chemical series since not only is it composed of elements with the same oxidation state and decreasing ionic radius but also they can be mimicked by the lanthanide(III) ion, $\mathrm{Ln}^{3+}$, series, which is placed on the row above in the periodic table. From a chemical point of view, $\mathrm{Ln}^{3+}$ are $4 f$ elements, while $\mathrm{An}^{3+}$ are $5 f$ ones. Many of their properties should be similar, and an interesting (and important) one is how they behave in liquid water. ${ }^{2,3}$ Note that for many reasons, performing experiments on $\mathrm{Ln}^{3+}$ instead of $\mathrm{An}^{3+}$ is more practical since, for example, $\mathrm{Ln}^{3+}$ are not radioactive and they can be obtained more easily (in particular for the heaviest ones).

Investigations of $\mathrm{An}^{3+}$ ions in liquid water were thus performed in recent years by a number of experimental and theoretical studies. ${ }^{2,4-18}$ Note that experimental data are mainly

$\overline{{ }^{a)} \text { Electronic mail: riccardo.spezia@univ-evry.fr }}$ present from $\mathrm{U}^{3+}$ to $\mathrm{Cf}^{3+}$, while for $\mathrm{Es}^{3+}$, only a diffusion coefficient value is reported. ${ }^{19}$ A particularly useful theoretical method aimed at understanding water structure and dynamics around these ions (and thus characterizing their hydration behavior) is molecular dynamics (MD). To this end, the authors have studied some $\mathrm{An}^{3+}$ ions by developing specific force fields, and particularly important is the use of polarizable force fields, as done in most of these studies..$^{9,11,13,15}$ Note that for highly charged ions, the explicit polarization of water molecules is important since such highly charged ions strongly impact on first shell molecules. ${ }^{20-22}$ To develop these force fields, quantum chemical calculations are often carried out, and parameters are fitted to obtain relevant properties (energies and structures) from $a b$ initio calculations. In the case of the $\mathrm{An}^{3+}$ series, this can be particularly difficult since many ions are open shell systems and high level calculations are needed. This is why one of the most studied ions is $\mathrm{Cm}^{3+}$ which has a half-filled $5 f$ shell. An alternative approach was adopted by us in the last few years on $\mathrm{Ln}^{3+}$ and $\mathrm{An}^{3+}$ ions based on the ionic radius behavior across the series, and polarizable potentials were successfully developed. ${ }^{23-25}$ In the case of $\mathrm{An}^{3+}$, originally only ionic radii in solid phase were available: they are Shannon's radii that change with the coordination number $(\mathrm{CN}),{ }^{26}$ and thus slightly different parameters (and associated results) were obtained for $\mathrm{CN}=8$ and $\mathrm{CN}=9,{ }^{25}$ the two CNs' characteristic of these ions in liquid water. ${ }^{2}$ Recently, D'Angelo and co-workers have obtained effective ionic radii in liquid water, ${ }^{27}$ so that it is now possible to obtain definitive and unambiguous parameters for each element. This was done in the present work. Furthermore, following a recent study of Migliorati et al. on $\mathrm{Ln}^{3+}$ ions ${ }^{28}$ and having in hands these 
effective ionic radii, we also developed a simpler LennardJones (LJ), non-polarizable potential. This force field, which in principle is less accurate than a polarizable one, can be easily extended to different systems since for each ion, it provides LJ parameters which can be used with combination rules to study other systems containing actinides, from proteins,${ }^{29-33}$ to mixtures, ${ }^{34}$ to organic, ${ }^{35,36}$ biphasic, ${ }^{37}$ or ionic liquids. ${ }^{38-40}$ We have thus compared results of this simple LJ force field with the polarizable one in order to open the possibility of also using simple LJ models, particularly useful for multi-component systems and/or to avoid specific parameterization. In this way, we were able to directly compare microscopic details for which experiments are lacking, especially as concerns the exchange dynamics of first shell water molecules. Results of both force fields are also directly compared to X-ray absorption data, in particular, through a direct comparison of the extended X-ray absorption fine structure (EXAFS) signals to assess the structural properties that are the starting point of more detailed analysis, such as the exchange dynamics.

\section{FORCE FIELD DEVELOPMENT}

\section{A. Polarizable force field}

For the polarizable force field, we used the same functional form of our previous works, ${ }^{23,24,27,41,42}$ to treat both electrostatic and non-electrostatic terms. The electrostatic is treated as the sum of a charge-charge interaction plus the interaction of a point charge with an induced dipole

$$
\begin{aligned}
V_{\text {elec }}= & \frac{1}{2} \sum_{i, j, i \neq j}\left[\frac{q_{i} q_{j}}{r_{i j}}+\frac{1}{r_{i j}^{3}}\left(-q_{i} \vec{p}_{j}+q_{j} \vec{p}_{i}\right) \cdot \vec{r}_{i j}\right. \\
& \left.+\vec{p}_{i} \cdot \underline{T}_{i j} \cdot \vec{p}_{j}\right]+\frac{1}{2} \sum_{i} \vec{p}_{i} \cdot\left(\underline{\alpha}_{i}\right)^{-1} \cdot \vec{p}_{j} .
\end{aligned}
$$

We used the Thole model, ${ }^{43}$ in which each site $i$ carries a permanent charge, $q_{i}$, and an induced dipole, $\vec{p}_{i}$, associated with an isotropic atomic polarizability tensor, $\underline{\alpha}_{i}, \vec{r}_{i j}=\vec{r}_{j}-\vec{r}_{i}$,

$$
\begin{aligned}
\underline{T}_{i j} & =\frac{1}{r_{i j}^{3}}\left(\underline{1}-3 \frac{A_{i j}}{r_{i j}^{2}}\right), \\
\underline{A}_{i j} & =\left[\begin{array}{ccc}
x_{i j}^{2} & x_{i j} y_{i j} & x_{i j} z_{i j} \\
y_{i j} x_{i j} & y_{i j}^{2} & y_{i j} x_{i j} \\
z_{i j} x_{i j} & z_{i j} y_{i j} & z_{i j}^{2}
\end{array}\right], \\
x_{i j} & =\left(x_{j}-x_{i}\right), \\
y_{i j} & =\left(y_{j}-y_{i}\right), \\
z_{i j} & =\left(z_{j}-z_{i}\right),
\end{aligned}
$$

and the last term, $\frac{1}{2} \sum_{i} \vec{p}_{i} \cdot\left(\underline{\alpha}_{i}\right)^{-1} \cdot \vec{p}_{j}$, is the polarization energy. Following the Thole model, a continuous screening function is used to avoid the so-called polarization catastrophe,

$$
\phi^{s}\left(r_{i j}\right)=\frac{1}{r_{i j}}\left[1-\left(1+\frac{a u}{2}\right) e^{-a u}\right],
$$

with $u=r_{i j} /\left(\alpha_{i} \alpha_{j}\right)^{1 / 6}$ and the parameter $a$ is $2.1304 \AA^{-1}$. This value was optimized and discussed in previous works. ${ }^{41,44}$
For a given configuration, the induced dipole on the atom $i$ is given by solving

$$
\vec{p}_{i}=\underline{\alpha}_{i} \cdot\left(\vec{E}_{i}+\sum_{i \neq j} \underline{T}_{i j} \cdot \vec{p}_{j}\right),
$$

where $\vec{E}_{i}$ is the sum of any applied external electric field and of the electrostatic field created by the permanent charges, $q_{j}$, on the induced dipole $\vec{p}_{i}$ and $\underline{T}_{i j} \cdot \vec{p}_{j}$ is the electric field created by the induced dipoles $\vec{p}_{j}$ on $\vec{p}_{i}$. To obtain one induced dipole, it is necessary to know all the induced dipoles, and this leads to a self-consistent problem which is solved iteratively at the beginning of the simulation. Since this step is time consuming, we used the extended Lagrangian approach ${ }^{45}$ to obtain induced dipoles along the trajectory.

For the non-electrostatic term, we used a simple LJ potential for water-water interactions, while for $\mathrm{Ln}-\mathrm{O}$ interactions, we have employed a Buckingham potential

$$
V_{n o n-e l e c}^{L n-O}\left(r_{i j}\right)=\sum_{i, j, i \neq j} A_{i j} e^{-B_{i j} r_{i j}}-\frac{C_{i j}}{r_{i j}^{6}} .
$$

For water, we used the same parameters, for both electrostatic and LJ potentials, employed successfully in the past to study hydration of very heavy metals..$^{23,24,27,41,42}$ They correspond to a modified polarizable TIP3P water model ${ }^{46,47}$ in which charges are rescaled to reproduce the dipole moment of liquid water. Note that within this simple water model, we reproduced correctly the exchange dynamics of water molecules in the first shell of ions and ionic diffusion for lanthanides(III) in water. ${ }^{22,23,48}$

For actinides, we proposed some years ago $A, B$, and $C$ parameters based on the Shannon ionic radii obtained in crystals $^{26}$ and specific to different coordination numbers. ${ }^{25}$ Recently, D'Angelo and co-workers have proposed by accurate EXAFS analysis new effective ionic radii for actinides in water. ${ }^{27}$ We have thus reparametrized the Buckingham potential accordingly. In particular, we have used the extrapolation procedure successfully employed in the past to reparameterize the lanthanide(III) force field on their corresponding effective ionic radii in water. ${ }^{42}$ Here, we have done the same for actinides as follows. Given the starting values for $B$ and $C$ parameters, called $B_{r e f}$ and $C_{r e f}$, corresponding to a $3+$ ion with a formal ionic radius $I R_{\text {ref }}$, it is possible to extrapolate new values for different values of ionic radii following

$$
\begin{aligned}
& B_{\text {new }}=B_{\text {ref }}-\kappa \Delta I R, \\
& C_{\text {new }}=C_{\text {ref }}+\gamma \Delta I R,
\end{aligned}
$$

where $\Delta I R=I R_{\text {new }}-I R_{\text {ref }}$, and $\kappa$ and $\gamma$ are the proportionality factors being $\kappa=1 \AA^{-2}$ and $\gamma=5.1211 \mathrm{~kJ} \mathrm{~mol}^{-1} \AA^{5}$. The justification of the expressions and of the parameters used is detailed in Ref. 25. As reference values, we have considered $\mathrm{U}^{3+}$ in solid which has a Shannon radius of $1.213 \AA$, and $B_{r e f}=3.483 \AA^{-1}$ and $C_{r e f}=3.7464 \times 10^{4} \mathrm{~kJ} \mathrm{~mol}^{-1} \AA^{6}$. Using the difference between this reference ionic radius and the radii for $\mathrm{An}^{3+}$ in water and the simple equations (8) and (9), the new parameters are easily obtained. For atomic polarizabilities, we used calculated data from our previous work. ${ }^{25}$ All the parameters for $\mathrm{An}^{3+}$ ions are reported in Table I. 
TABLE I. Force field parameters used in the polarizable MD simulations: $B$ in $\AA^{-1}, C$ in kJ mol ${ }^{-1} \AA^{6}$, polarizability, $\alpha$ in $\AA^{-1}$, and ionic radii, IR, in $\AA$. $\sigma_{A n}(\AA)$ are the parameters of the Lennard-Jones potentials used in the non-polarizable MD simulations, while $\sigma_{A n A n}(\AA)$ are the Lennard-Jones parameters describing generic An-An interactions, which have been derived from the ion-solvent parameters using Lorentz-Berthelot combination rules.

\begin{tabular}{lcccccc}
\hline \hline Ion & $\mathrm{B}$ & $\mathrm{C} / 10^{4}$ & $\alpha$ & $\mathrm{IR}$ & $\sigma_{A n}$ & $\sigma_{A n A n}$ \\
\hline $\mathrm{U}^{3+}$ & 3.519 & 3.5237 & 1.846 & 1.177 & 3.230 & 3.294 \\
$\mathrm{~Np}^{3+}$ & 3.537 & 3.4316 & 1.633 & 1.159 & 3.201 & 3.236 \\
$\mathrm{Pu}^{3+}$ & 3.556 & 3.3342 & 1.486 & 1.140 & 3.171 & 3.176 \\
$\mathrm{Am}^{3+}$ & 3.574 & 3.2421 & 1.363 & 1.122 & 3.142 & 3.118 \\
$\mathrm{Cm}^{3+}$ & 3.591 & 3.1550 & 1.238 & 1.105 & 3.115 & 3.064 \\
$\mathrm{Bk}^{3+}$ & 3.607 & 3.0731 & 1.197 & 1.089 & 3.089 & 3.012 \\
$\mathrm{Cf}^{3+}$ & 3.624 & 2.9860 & 1.166 & 1.072 & 3.062 & 2.958 \\
\hline \hline
\end{tabular}

\section{B. Lennard-Jones force field}

In addition to the use of a polarizable force field, we have investigated the hydration properties of actinide ions in aqueous solution by carrying out MD simulations without explicit polarization. In this case, the electrostatic interactions were modeled by including charge-charge Coulombic interactions only, corresponding to the first term of Eq. (1). For water, the extended simple point charge (SPC/E) model has been employed, as it has been successfully used in the literature to study metal ion hydration. ${ }^{49-53}$ van der Waals interactions between the $\mathrm{An}^{3+}$ ion and the oxygen atoms of water molecules have been described using a LJ potential

$$
V_{L J}^{A n-O}\left(r_{i j}\right)=\sum_{i, j, i \neq j} 4 \epsilon_{i j}\left[\left(\frac{\sigma_{i j}}{r_{i j}}\right)^{12}-\left(\frac{\sigma_{i j}}{r_{i j}}\right)^{6}\right] .
$$

The $\sigma$ and $\epsilon$ LJ parameters have been refined based on the results of a recent combined MD-EXAFS investigation of the hydration properties of $\mathrm{Ln}^{3+}$ ions in water. ${ }^{28} \mathrm{In}$ that study, $\mathrm{LJ}$ parameters describing $\mathrm{Ln}^{3+}$-water interactions have been developed for the whole lanthanide series, by directly comparing the hydration structure obtained from MD simulations of $\mathrm{Ln}^{3+}$ aqueous solutions with the EXAFS experimental data. ${ }^{28}$ Here, we have used the same value of the $\epsilon$ parameter refined for $\mathrm{Ln}^{3+}$ ions $(0.4308 \mathrm{~kJ} / \mathrm{mol})$, while for $\sigma$, we have adopted the following extrapolation procedure. In analogy with the reparametrization of $B$ and $C$ of the polarizable force field described above, the refinement of $\sigma$ values for the $\mathrm{An}^{3+}$ ions $\left(\sigma_{A n}\right)$ was based on the values of the $\mathrm{An}^{3+}$ effective ionic radii proposed by D'Angelo and co-workers in Ref. 27. In particular, $\sigma_{A n}$ has been calculated as follows:

$$
\sigma_{A n}=\sigma_{\widetilde{L n}} \frac{B_{A n}}{B_{\widetilde{L n}}},
$$

where $\widetilde{L n}$ represents a hypothetical $\mathrm{Ln}^{3+}$ ion with the same ionic radius as the given $\mathrm{An}^{3+} . B_{A n}$ values are those reported in Table I, while $B_{\widetilde{L n}}$ has been calculated by applying Eq. (8) and starting from the $B$ value of the $\mathrm{Nd}^{3+}$ ion,

$$
B_{\widetilde{L n}}=B_{N d}-\left(I R_{\widetilde{L n}}-I R_{N d}\right)
$$

where $B_{N d}$ and $I R_{N d}$ were taken from Ref. 42 . On the other hand, $\sigma_{\widetilde{L n}}$ has been obtained starting from the $\sigma_{L n}$ parameters of $\mathrm{Ln}^{3+}$ in water developed in Ref. 28, by plotting the $\sigma_{L n}$

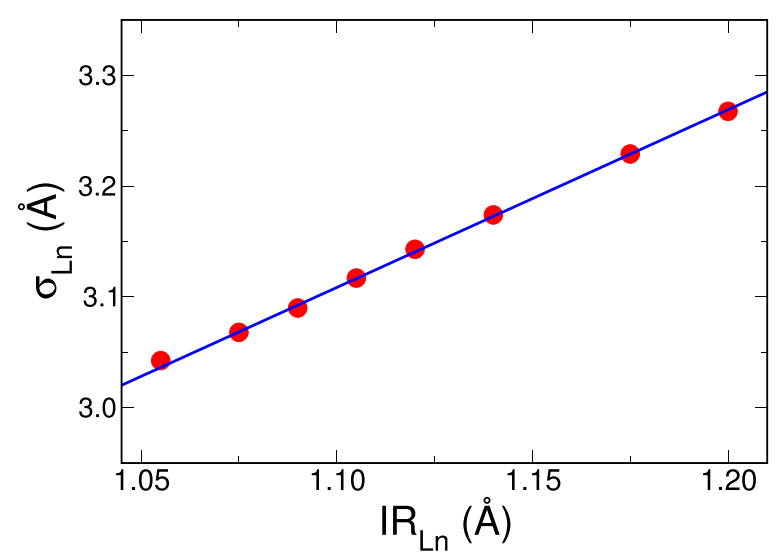

FIG. 1. Lennard-Jones $\sigma_{L n}$ parameters developed in Ref. 28 for $\mathrm{Ln}^{3+}$ ions in water plotted as a function of the $\mathrm{Ln}^{3+}$ ionic radii.

values as a function of the $\mathrm{Ln}^{3+}$ ionic radii (Fig. 1). As it can be seen, the $\sigma_{L n}$ values can be fitted by a linear expression

$$
\sigma_{L n}=1.3433+1.6047 I R_{L n} .
$$

By inserting in the equation the ionic radius of a given $\mathrm{An}^{3+}$ ion, we can obtain the $\sigma_{\widetilde{L n}}$ value of a hypothetical $\mathrm{Ln}^{3+}$ ion with the same ionic radius. The $\sigma_{A n}$ parameter values obtained by applying this procedure and used in the MD simulations of $\mathrm{An}^{3+}$ aqueous solutions are reported in Table I.

In order to use these $\mathrm{LJ}$ parameters in MD simulations of other $\mathrm{An}^{3+}$-containing systems, it is useful to extrapolate generic parameters describing the An-An pair interactions $\left(\sigma_{A n A n}\right.$ and $\left.\epsilon_{A n A n}\right)$ by using Lorentz-Berthelot combination rules, starting from the $\mathrm{LJ}$ parameter values for the $\mathrm{An}-\mathrm{O}$ interactions and for the $\mathrm{O}-\mathrm{O}$ interactions as described in the SPC/E water model. ${ }^{54}$ The resulting $\epsilon_{A n A n}$ value is $0.2855 \mathrm{~kJ} / \mathrm{mol}$ for all of the $\mathrm{An}^{3+}$ ions, while the $\sigma_{A n A n}$ values are reported in Table I. Note that the $\sigma_{A n A n}$ and $\epsilon_{A n A n}$ values can be combined with force field parameters available in the literature in order to obtain pair potentials involving $\mathrm{An}^{3+}$ ions in other disordered systems.

\section{COMPUTATIONAL DETAILS}

\section{A. Polarizable simulations}

MD simulations using the polarizable force field have been carried out using the MDVRY code. ${ }^{44}$ Simulation systems consist of one ion immersed in cubic boxes composed of 216 water molecules, with box edges of $18.64 \AA$, which gives the correct density as reported previously. ${ }^{55,56}$ Periodic boundary conditions were applied to each simulation box in order to mimic bulk conditions. Long-range interactions were calculated by using the smooth particle mesh Ewald method. ${ }^{57}$ Simulations were performed using a velocity-Verlet-based multiple time step in the microcanonical NVE ensemble with our own developed code MDVRY. ${ }^{44}$ The equations of motion were numerically integrated using a $1 \mathrm{fs}$ time step. The system was equilibrated at $300 \mathrm{~K}$ for $2 \mathrm{ps}$. In order to assure the stability of dipole dynamics, we performed for each ion six sets of simulations of $3 \mathrm{~ns}$ each, where the dipoles were re-optimized via the self-consistent procedure at the beginning of each set 
and then, after an equilibration time of $2 \mathrm{ps}$, they were let to evolve using the extended Lagrangian method (see details in Sec. II A). In this way, we obtained a total of $18 \mathrm{~ns}$ to calculate average properties.

\section{B. Non-polarizable simulations}

MD simulations of $\mathrm{An}^{3+}$ aqueous solutions using the nonpolarizable force field have been carried out by means of the GROMACS package. ${ }^{58}$ The systems were composed of one $\mathrm{An}^{3+}$ ion and 819 water molecules placed in a cubic box, with box edges of $29.09 \AA$ and replicated using periodic boundary conditions. Note that the number density of oxygen atoms in the polarizable and non-polarizable simulation boxes is identical $\left(0.0333 \AA^{-3}\right)$. The simulations were performed in the NVT ensemble at $300 \mathrm{~K}$ using the Nosé-Hoover thermostat, ${ }^{59,60}$ with a relaxation constant of 0.5 ps. A cutoff of $9 \AA$ was adopted for the nonbonded interactions, and long-range electrostatic interactions were treated with the particle mesh Ewald method. ${ }^{57}$ All of the simulations were carried out for $18 \mathrm{~ns}$ with a time step of $1 \mathrm{fs}$, after an equilibration run of 3 ns.

\section{ANALYSIS METHODS}

\section{A. Structural properties}

From equilibrated trajectories, structural properties are characterized first by means of radial distribution functions, $g(r)$ 's. In particular, as in the present case, the arrangement of water molecules around the solute ion is the important quantity, and An-O and An-H $g(r)$ 's are calculated. From them, it is possible to identify the first hydration shell and thus a cutoff radius to calculate for each snapshot the number of water molecules in the first shell. An important structural quantity is the distance between water molecules and the central ion, and in this framework, there are two characteristic quantities: the maximum of $g(r)$ and the average value. These two values are identical only for a symmetric distribution, which is not the case in general for ion hydration, and their difference is an indirect measure of the asymmetry of the distribution. It is important to note that literature data can report either the maximum or the average distance and thus it is important to report both to make accurate comparisons. The $\mathrm{CN}$ values can be obtained by the integral of $g(r)$ or, when a clear distinction between two shells is obtained, by defining a cutoff radius and then calculating the number of water molecules which are inside this radius at each step. In this last case, it is possible to attribute an uncertainty to the average CN.

\section{B. Dynamical properties}

Dynamics of water molecules around a solute ion is calculated to identify a mean residence time (MRT). Often the Impey method is used, ${ }^{61}$ but this can be safely done only for systems in which there are several exchanges, and thus the correlation function, which is the basic calculated quantity, goes to zero in the simulated time scale. In the present case, in particular for some ions, the exchange dynamics is very slow, and thus the correlation functions do not go to zero and/or they do not show a clear single exponential behavior in the time scale studied. We have thus used for all the systems the direct method, ${ }^{62}$ in which we count the number of molecules that leave or enter the ion first hydration shell and that after coming in (or out) does not go back out (or in) before a given time, $\mathrm{t}^{*}$. This $\mathrm{t}^{*}$ has the same meaning as the usual $\mathrm{t}^{*}$ used in the Impey method. The MRT is then obtained by the simple expression

$$
M R T=\langle C N\rangle * t_{\text {sim }} / N_{\text {exch }},
$$

where $\langle C N\rangle$ is the average coordination number, $t_{\text {sim }}$ is the total simulation time, and $N_{\text {exch }}$ is the number of exchanges. The direct count method suffers less convergence problems: in fact in the Impey method, the fitting of clearly non-exponential correlation functions with a single exponential is too arbitrary, providing unreliable results, while in the direct count method, one has just to count the exchange events. Of course, if the solvent exchange is badly converged, the results will not be converged as well. At this aim, we have evaluated the uncertainties in MRT, by propagating uncertainties in $\langle C N\rangle$ and $N_{\text {exch }}$ on the different trajectories, in order to provide a quantification of the degrees of convergence.

\section{EXAFS signal reconstruction}

The $\mathrm{L}_{3}$-edge $\mathrm{X}$-ray absorption spectra of $\mathrm{An}^{3+}$ ions in aqueous solution used in this work were collected by different research groups that provided us with the raw data as explained in Ref. 27. Experimental details on sample preparation and EXAFS data acquisition can be found in the original works. $^{27}$

The EXAFS data were analyzed with a modified version of the GNXAS program that calculates phase shifts and theoretical signals also for actinides. ${ }^{63-66}$ Phase shifts have been calculated using muffin-tin potentials and advanced models for the exchange-correlation self-energy. Several investigations have shown that in the case of aqueous solutions rather than using the usual discrete form of the EXAFS equation, the $\chi(k)$ signal is modelled as a function of the radial distribution function $g(r)$ as ${ }^{67-71}$

$$
\chi(k)=\int_{0}^{\infty} d r 4 \pi \rho r^{2} g(r) A(k, r) \sin [2 k r+\phi(k, r)],
$$

where $A(k, r)$ and $\phi(k, r)$ are the amplitude and phase functions, respectively, and $\rho$ is the density of the scattering atoms. The EXAFS theoretical signals are calculated by introducing in Eq. (15) An-O and An-H $g(r)$ 's obtained from MD simulations, and the comparison between the theoretical and experimental spectra allows the reliability of the potential functions used in the simulations to be checked. Least-squares fits of the EXAFS raw experimental data have been carried out and two non-structural parameters have been optimized, namely, $E_{0}$ (core ionization threshold energy) and $S_{0}^{2}$.

\section{RESULTS}

\section{A. Aqua ions structural properties}

An-O $g(r)$ 's obtained from both polarizable (labeled here and hereafter Pol-MD) and non-polarizable LJ based (labeled 


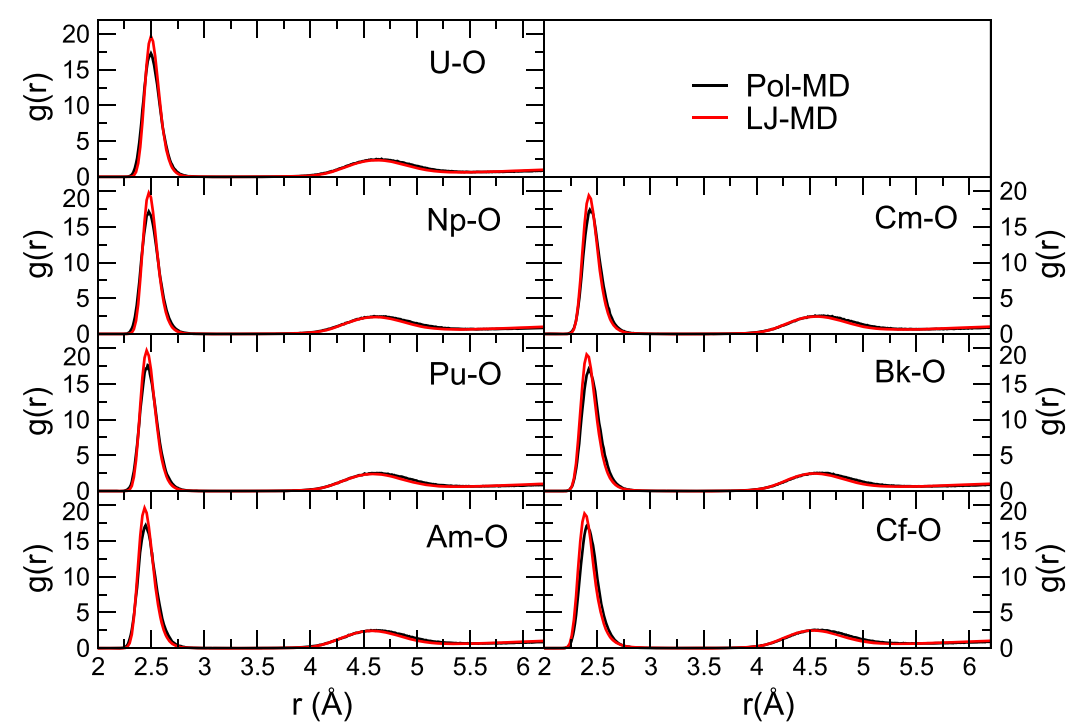

FIG. 2. An-O radial distribution functions obtained from polarizable and Lennard-Jones simulations, PolMD and LJ-MD, respectively.
LJ-MD) MD simulations are reported in Fig. 2. As already known from previous studies, ${ }^{2,25,27} \mathrm{An}^{3+}$ ions strongly structurate water molecules forming a well-defined first hydration shell. Also, a second shell is clearly visible from Fig. 2, as already noticed. ${ }^{25}$ Comparing the Pol-MD with LJ-MD, we can point out that the latter simulations have the tendency to structurate more the water molecules around the ion, as evident from the higher intensity of the LJ-MD $g(r)$ first peaks. The distances obtained by Pol-MD and LJ-MD are very similar for light actinides, while LJ-MD simulations show slightly shorter distances for heavier ions. More structural details are obtained by a careful analysis of $g(r)$ 's, from which the distances corresponding to the maximum can be extracted (values are reported in Table II). From these $g(r)$ 's, it is possible to easily define as first shell water molecules those within $3.2 \AA$ from the central ion. Average distances and CNs are reported in Table II. From this table, it is possible to quantify the small differences of An-O distances between Pol-MD and LJ-MD results. Also, CNs show small differences, in particular, for $\mathrm{Cf}^{3+}$. We will examine these differences in more detail by looking to the exchange dynamics of water molecules in the first shell in Sec. V B. We first compare our results with literature data and then with the experimental EXAFS signals in order to assess the validity of the two new force fields developed.

MD simulations reported for $\mathrm{An}^{3+}$ ions are scarce (most of them refer to $\mathrm{Cm}^{3+}$ and $\mathrm{Cf}^{3+}$ ), and different authors have developed in the last few years different force fields (generally polarizable) for these ions. ${ }^{9}, 12-16$ In Table II, we report these results, as well as some density functional theory (DFT)-based and Monte Carlo (MC) simulations. ${ }^{10,14}$ For $\mathrm{Cm}^{3+}$, previous simulations report longer distances and a $\mathrm{CN}$ generally of 9, with the exception of the AMOEBA force field ${ }^{15}$ which provides distances similar to our results for both $\mathrm{Cm}^{3+}$ and $\mathrm{Am}^{3+}$. As we will see, the experimental distances are in agreement with those obtained by our and AMOEBA force fields. For $\mathrm{Cf}^{3+}$, simulations by the group of Sanchez-Marcos report distances in the 2.43-2.47 $\AA$ range, not very different from our distances, but a $\mathrm{CN}$ of 8 , while our simulations report $\mathrm{CN}=9$ both with Pol-MD and LJ-MD. CN is a difficult quantity to be determined by EXAFS with good accuracy, but the good agreement with the EXAFS experimental data (as we will discuss in the following) is an indication that the structure reported as $\mathrm{CN}=9$ is dominant. Finally, DFT-based MD simulations are also reported for $\mathrm{Pu}^{3+}$, providing longer distances, while it seems that the 8-fold structure is more stable even if the 9-fold one cannot be excluded (DFT-based trajectories are too limited in time to grab the slow water exchange dynamics). ${ }^{16}$

EXAFS experiments were performed by different authors, ${ }^{3-7,10,72}$ and recently, D'Angelo and co-workers have collected and analyzed them using a single-shell model that makes comparison with MD simulations straightforward. ${ }^{27}$ In particular, the average structural values are directly comparable with simulation average An-O distances. These data are reported in Table II and they can be compared with our results: our simulations are in almost perfect agreement with these values, for both Pol-MD and LJ-MD models.

We should notice that for heavier ions, LJ-MD distances are about $0.02 \AA$ shorter than the Pol-MD ones, and the EXAFS distances are also $0.02 \AA$ shorter than the Pol-MD ones. This difference is very small, of the order of magnitude of the experimental uncertainty in distance, and to better understand the reliability of our simulations, we made a direct comparison with the EXAFS experimental data. Starting from the Pol-MD and LJ-MD An-O and An-H $g(r)$ 's, we calculated for each $\mathrm{An}^{3+}$ ion a total $\chi(k)$ theoretical signal using Eq. (15), and we compared it with the experimental signal. Minimization procedures were carried out in the range $\mathrm{k}=3.4-10.1 \AA^{-1}$, and during the analysis, the structural contributions were kept fixed. In this way, the first hydration shell structure derived from the MD simulations can be directly compared with the experimental data, and the reliability of the force fields used in the simulations can be assessed. The results of these analyses are shown in Figs. 3 and 4 for Pol-MD and LJ-MD, respectively. In particular, Figs. 3 and 4 show the comparison of the EXAFS experimental spectra with the total $\chi(k)$ theoretical signals calculated from MD $g(r)$ 's for the actinide series together with the residual curves. The agreement between the experimental and theoretical data is very good in all cases for 
TABLE II. Structural results obtained from MD simulations of $\mathrm{An}^{3+}$ in water: we report $\mathrm{r}_{A n O}$ and $\mathrm{r}_{A n H}$ as the maximum of $g(r)$ 's (values in $\AA$ ), the average An-O distance, $\left\langle\mathrm{r}_{A n O}\right\rangle$, and the average coordinate number, $\langle\mathrm{CN}\rangle$. For these two last average quantities, we also report uncertainty as a fluctuation of the mean. As experimental data, we report the EXAFS average distance obtained by using a single-shell model (see the original Ref. 27 for details). When available, we report also other simulation results.

\begin{tabular}{|c|c|c|c|c|c|}
\hline Ion & Method & $\mathrm{r}_{A n O}$ & $\mathrm{r}_{A n H}$ & $\left\langle\mathrm{r}_{A n O}\right\rangle$ & $\langle\mathrm{CN}\rangle$ \\
\hline \multirow[t]{3}{*}{$\mathrm{U}^{3+}$} & Pol-MD & 2.50 & 3.15 & $2.53 \pm 0.02$ & $9.00 \pm 0.06$ \\
\hline & LJ-MD & 2.50 & 3.18 & $2.53 \pm 0.02$ & $9.01 \pm 0.09$ \\
\hline & EXAFS & & & 2.527 & 9 \\
\hline \multirow[t]{3}{*}{$\mathrm{Np}^{3+}$} & Pol-MD & 2.48 & 3.14 & $2.51 \pm 0.02$ & $9.00 \pm 0.06$ \\
\hline & LJ-MD & 2.48 & 3.16 & $2.51 \pm 0.02$ & $9.00 \pm 0.04$ \\
\hline & EXAFS & & & 2.509 & 9 \\
\hline \multirow[t]{5}{*}{$\mathrm{Pu}^{3+}$} & Pol-MD & 2.47 & 3.13 & $2.50 \pm 0.02$ & $9.00 \pm 0.05$ \\
\hline & LJ-MD & 2.46 & 3.14 & $2.49 \pm 0.02$ & $9.00 \pm 0.03$ \\
\hline & EXAFS & & & 2.490 & 9 \\
\hline & $\mathrm{MD}^{\mathrm{a}}$ & 2.56 & & & 9 \\
\hline & $\mathrm{MD}^{\mathrm{a}}$ & 2.53 & & & 8 \\
\hline \multirow[t]{4}{*}{$\mathrm{Am}^{3+}$} & Pol-MD & 2.45 & 3.11 & $2.49 \pm 0.02$ & $8.99 \pm 0.08$ \\
\hline & LJ-MD & 2.44 & 3.12 & $2.47 \pm 0.02$ & $9.00 \pm 0.03$ \\
\hline & EXAFS & & & 2.472 & 9 \\
\hline & $\mathrm{MD}^{\mathrm{b}}$ & 2.44 & & & 9 \\
\hline \multirow[t]{8}{*}{$\mathrm{Cm}^{3+}$} & Pol-MD & 2.43 & 3.11 & $2.47 \pm 0.02$ & $8.99 \pm 0.12$ \\
\hline & LJ-MD & 2.42 & 3.10 & $2.46 \pm 0.02$ & $9.00 \pm 0.04$ \\
\hline & EXAFS & & & 2.455 & 9 \\
\hline & $\mathrm{MD}^{\mathrm{b}}$ & 2.43 & & & 9 \\
\hline & $\mathrm{MD}^{\mathrm{c}}$ & 2.55 & & & 8.9 \\
\hline & $\mathrm{MD}^{\mathrm{d}}$ & 2.48 & & & 9 \\
\hline & $\mathrm{MD}^{\mathrm{e}}$ & $2.52-2.53$ & & & 9 \\
\hline & $\mathrm{MD}^{\mathrm{f}}$ & 2.49 & & & 8.6 \\
\hline \multirow[t]{3}{*}{$\mathrm{Bk}^{3+}$} & Pol-MD & 2.42 & 3.09 & $2.46 \pm 0.02$ & $8.96 \pm 0.20$ \\
\hline & LJ-MD & 2.40 & 3.09 & $2.44 \pm 0.02$ & $8.99 \pm 0.08$ \\
\hline & EXAFS & & & 2.439 & 9 \\
\hline \multirow[t]{5}{*}{$\mathrm{Cf}^{3+}$} & Pol-MD & 2.41 & 3.08 & $2.45 \pm 0.02$ & $8.91 \pm 0.28$ \\
\hline & LJ-MD & 2.39 & 3.08 & $2.43 \pm 0.02$ & $8.97 \pm 0.17$ \\
\hline & EXAFS & & & 2.422 & 9 \\
\hline & $\mathrm{MC}^{\mathrm{g}}$ & $2.43-2.47$ & & & 8 \\
\hline & $\mathrm{MD}^{\mathrm{h}}$ & 2.44 & & & 8 \\
\hline
\end{tabular}

${ }^{\mathrm{a} D F T}$-based MD (PBE functional) from Ref. 16.

${ }^{\mathrm{b}} \mathrm{MD}$ with AMOEBA polarizable force fields from Ref. 15

${ }^{\mathrm{c}}$ Polarizable MD from Ref. 9.

${ }^{\mathrm{d}}$ DFT-based MD (PBE functional) from Ref. 14.

${ }^{\mathrm{e}}$ Non-polarizable MD from Ref. 14.

${ }^{\mathrm{f}}$ Average values from the polarizable potential using different contributions in the interaction energy, from Ref. 13.

${ }^{\mathrm{g}}$ Monte Carlo simulations using a polarizable force field from Ref. 11.

${ }^{\mathrm{h}}$ Polarizable MD with the same force field as $(\mathrm{g})$, from Ref. 12.

both potentials, proving the reliability of the developed force fields.

Unfortunately, EXAFS is not able to directly provide a coordination number with high accuracy as shown in Refs. 27, 42, and 73. Our previous simulations have reported that the main coordination number of light actinides is nine, similarly to lanthanides. ${ }^{25,27}$ In MD simulations, however, the number of water molecules often fluctuates around the central ion, even in the case of highly charged cations like $\mathrm{An}^{3+}$, which strongly structurate the first shell solvent molecules. Thus, the observed non-integer coordination numbers in simulations are a result of the coexistence of different coordination motifs. In the present case, in addition to the 9-fold structure (which is the most relevant), we observed some $\mathrm{CN}=10$ (for lighter

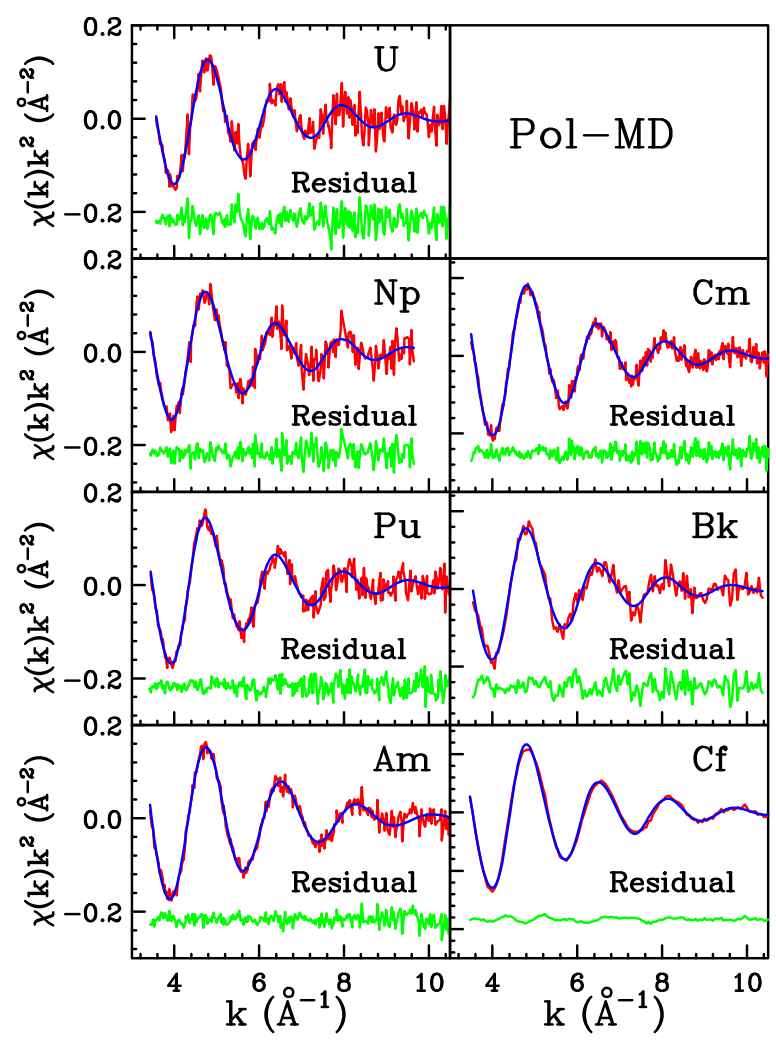

FIG. 3. Comparison between the $\mathrm{L}_{3}$-edge EXAFS experimental data (red line) of the $\mathrm{An}^{3+}$ ions in aqueous solution and the theoretical signal (blue line) calculated from An-O and An-H $g(r)$ 's obtained from the Pol-MD simulations. The residual curves are also shown (green line).

elements in the series) and $\mathrm{CN}=8$ (for heavier ones). The different populations of these three coordination numbers are reported in Table III as obtained for both Pol-MD and LJ-MD. The two methods provide similar results, where the LJ-MD simulations have the tendency to overestimate higher CNs: for light atoms, $\mathrm{CN}=10$ is slightly higher in LJ-MD than that obtained by Pol-MD, and for heavier ones, the $\mathrm{CN}=8$ coordination is less populated in LJ-MD than that obtained with Pol-MD.

\section{B. Water exchange dynamics}

As we have pointed out, the average coordination numbers result from the equilibrium between different values, even if in the elements investigated here (i.e., the $\mathrm{An}^{3+}$ ions for which EXAFS data are available), the $\mathrm{CN}=9$ structure is always dominant. We have followed the evolution in time of instantaneous CNs (i.e., the number of water molecules that are in the first hydration shell at each snapshot), as reported in Figs. 5-10. As shown by $\mathrm{CN}$ vs time for $\mathrm{U}^{3+}, \mathrm{Np}^{3+}$, and $\mathrm{Cf}^{3+}$ reported in figures, we have several exchanges: the structure is on average $\mathrm{CN}=9$, but it is possible that at different times, one molecule enters the first hydration shell, forming a 10-fold structure, or it leaves, forming a $\mathrm{CN}=8$ structure. This is a good indication that trajectories are equilibrated (on the contrary, if an average $\mathrm{CN}$ would result from a continuous portion of the trajectory with a given $\mathrm{CN}$ followed by a punctual $\mathrm{CN}$ modification, one should be questioned about the equilibration). The same behavior occurs for other elements not shown here for simplicity. We have chosen these three prototypical elements for the 


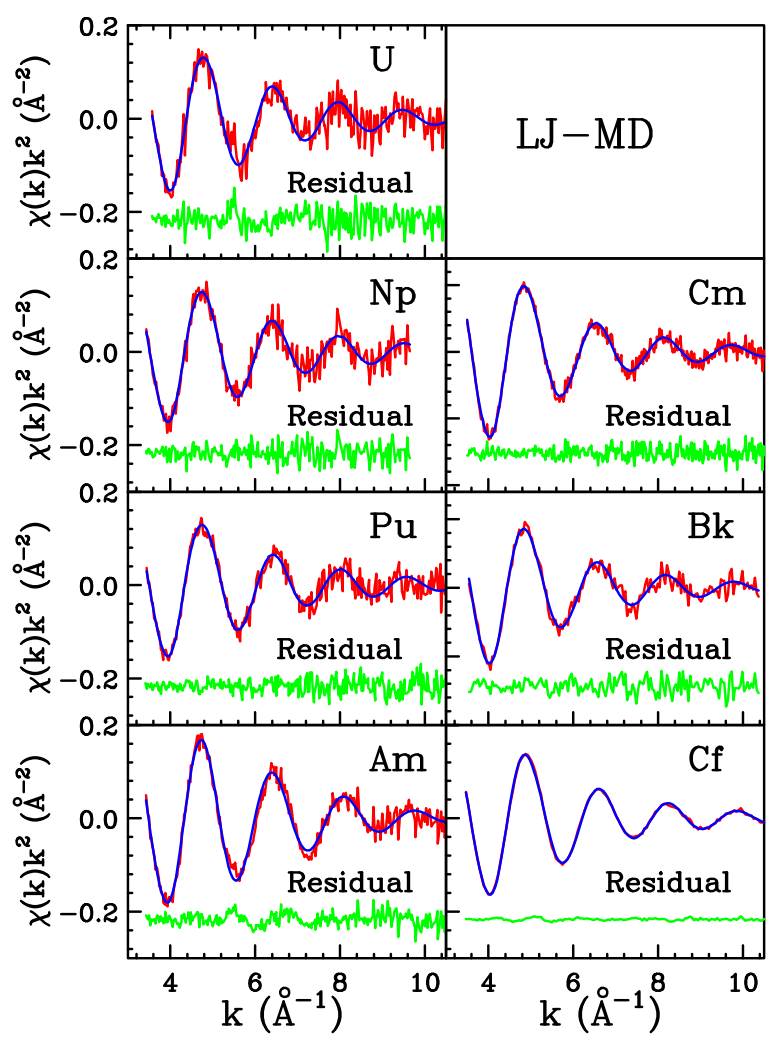

FIG. 4. Comparison between the $\mathrm{L}_{3}$-edge EXAFS experimental data (red line) of the $\mathrm{An}^{3+}$ ions in aqueous solution and the theoretical signal (blue line) calculated from An-O and An-H $g(r)$ 's obtained from the LJ-MD simulations. The residual curves are also shown (green line).

following reasons. In the case of $\mathrm{U}^{3+}$, we observe a difference between Pol-MD and LJ-MD: in Pol-MD, the occurrence of $\mathrm{CN}=10$ is relatively rare, as reflected by the small percentage of this $\mathrm{CN}$ in the average, while for LJ-MD, the number of exchanges is higher. Moving to the next element, $\mathrm{Np}^{3+}$, we have that for both force fields, the number of exchanges is small. We should notice this important phenomenon: a slight modification of the parameters $\left(\mathrm{U}^{3+}\right.$ and $\mathrm{Np}^{3+}$ are close and thus the $\sigma$ values are very similar) may produce a significant difference in water exchange dynamics. It is also important to
TABLE III. Percentages of different coordination numbers as obtained in polarizable (Pol-MD) and non-polarizable (LJ-MD) simulations.

\begin{tabular}{lcccc}
\hline \hline Ion & Method & $\% \mathrm{CN}=8$ & $\% \mathrm{CN}=9$ & $\% \mathrm{CN}=10$ \\
\hline $\mathrm{U}^{3+}$ & Pol-MD & 0.04 & 99.64 & 0.32 \\
& LJ-MD & 0.01 & 99.18 & 0.81 \\
$\mathrm{~Np}^{3+}$ & Pol-MD & 0.15 & 99.65 & 0.20 \\
& LJ-MD & 0.01 & 99.82 & 0.17 \\
$\mathrm{Pu}^{3+}$ & Pol-MD & 0.22 & 99.76 & 0.02 \\
& LJ-MD & 0.03 & 99.92 & 0.05 \\
$\mathrm{Am}^{3+}$ & Pol-MD & 0.74 & 99.25 & 0.01 \\
& LJ-MD & 0.06 & 99.93 & 0.01 \\
$\mathrm{Cm}^{3+}$ & Pol-MD & 1.53 & 98.47 & 0.00 \\
& LJ-MD & 0.17 & 99.82 & 0.01 \\
$\mathrm{Bk}^{3+}$ & Pol-MD & 4.25 & 95.75 & 0.00 \\
& LJ-MD & 0.61 & 99.38 & 0.01 \\
$\mathrm{Cf}^{3+}$ & Pol-MD & 8.86 & 91.14 & 0.00 \\
& LJ-MD & 2.92 & 97.08 & 0.00 \\
\hline \hline
\end{tabular}

remark that while differences in structural properties are very small, this can correspond to relatively important differences for the dynamical ones. In the case of $\mathrm{Cf}^{3+}$, the heaviest in the series studied here, there are many exchanges in both LJ-MD and Pol-MD. In this case starting from the dominant 9-fold structure, it is also possible to populate the 8-fold one, while the 10-fold structure population is negligible.

From $\mathrm{CN}$ time evolution, it is possible to obtain the mean residence time (MRT) for all the systems studied, with both force fields, as reported in Table IV. An important parameter used to evaluate the MRT is $t^{*}$ (see Sec. IV B for details). Here, we report results for two values of $t^{*}: 0.5 \mathrm{ps}$, which corresponds to the water-water $\mathrm{H}$-bond lifetime ${ }^{62}$ and which is often used in simulations, ${ }^{49,51,62,74-76}$ and $t^{*}=1 \mathrm{ps}$, which is the double of the previous value used to check the stability of MRT as a function of the parameter $t^{*}$. Results show that, as expected by the time evolution of $\mathrm{CN}$, the largest discrepancy between Pol-MD and LJ-MD is for $\mathrm{U}^{3+}$, in which the LJ-MD MRT value is lower than the one obtained from Pol-MD simulations. Furthermore, while for Pol-MD, the MRTs increase from $\mathrm{U}^{3+}$ to $\mathrm{Pu}^{3+}$ and then decrease, for $\mathrm{LJ}-\mathrm{MD}$, they increase
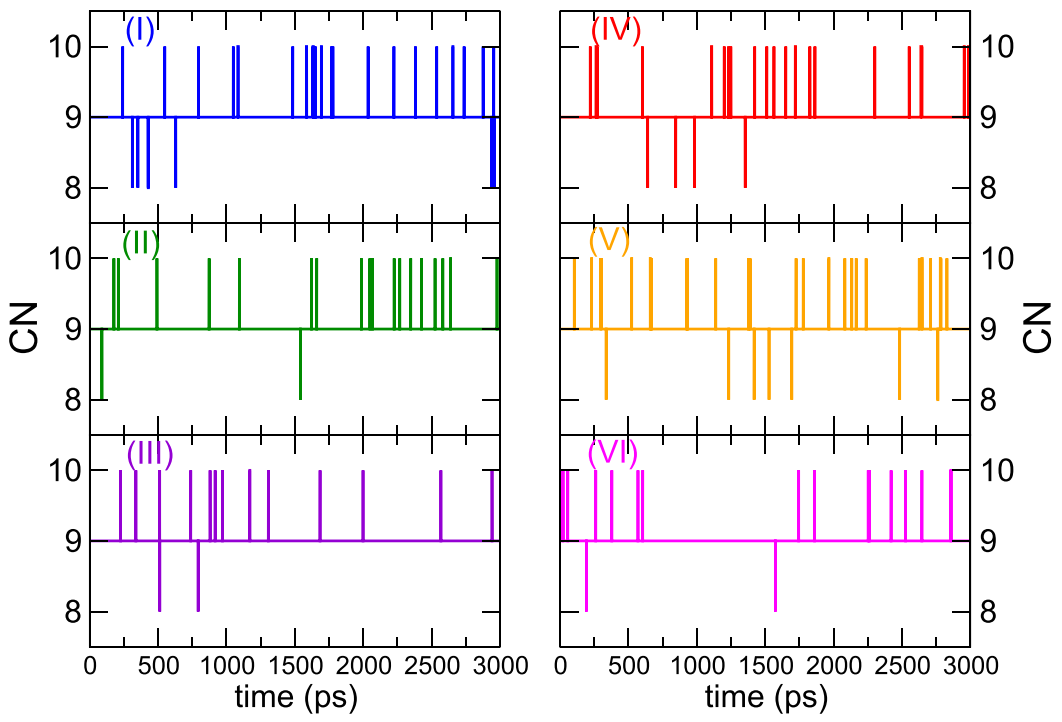

FIG. 5. First shell coordination number as a function of time for six independent molecular dynamics simulations of $\mathrm{U}^{3+}$ in aqueous solution as obtained from Pol-MD trajectories. 

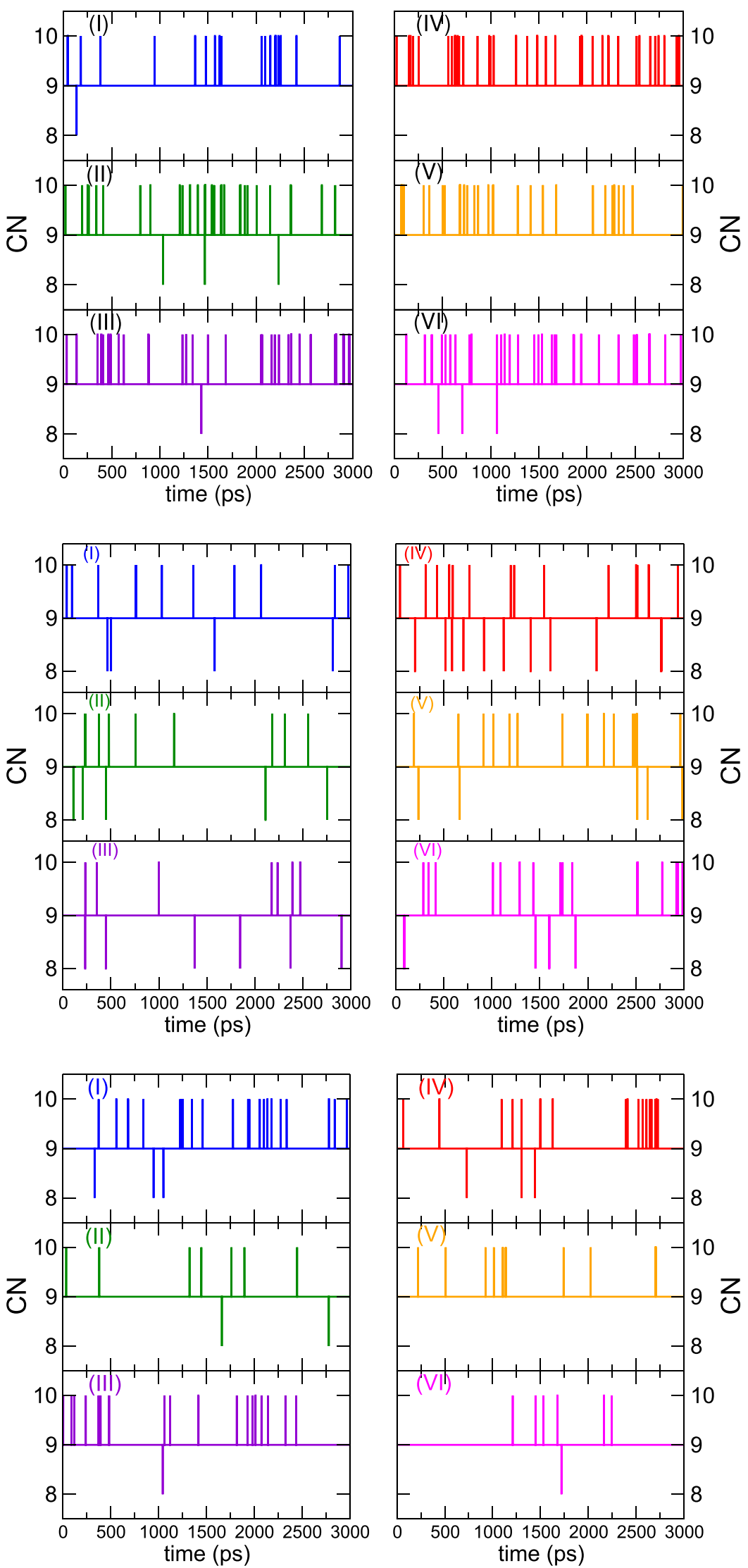

FIG. 6. First shell coordination number as a function of time as obtained from LJ-MD simulations of $\mathrm{U}^{3+}$. The 18 ns trajectory is divided into six to be compared with the equivalent Pol-MD results.
FIG. 7. First shell coordination number as a function of time for six independent molecular dynamics simulations of $\mathrm{Np}^{3+}$ in aqueous solution as obtained from Pol-MD trajectories.
FIG. 8. First shell coordination number as a function of time as obtained from LJ-MD simulations of $\mathrm{Np}^{3+}$. The $18 \mathrm{~ns}$ trajectory is divided into six to be compared with the equivalent Pol-MD results. up to $\mathrm{Am}^{3+}$ and then start decreasing. Results for the heaviest element, $\mathrm{Cf}^{3+}$, are, on the other hand, very similar between the two force fields. Interestingly, for light atoms, we have the largest dependence of MRT on $\mathrm{t}^{*}$ : this reflects the low number of exchanges and more, in particular, the fact that once a water molecule leaves or enters the first hydration shell, this process is not always fully equilibrated for light elements. On the other hand, for heavy $\mathrm{An}^{3+}$ ions, or more in general for elements 

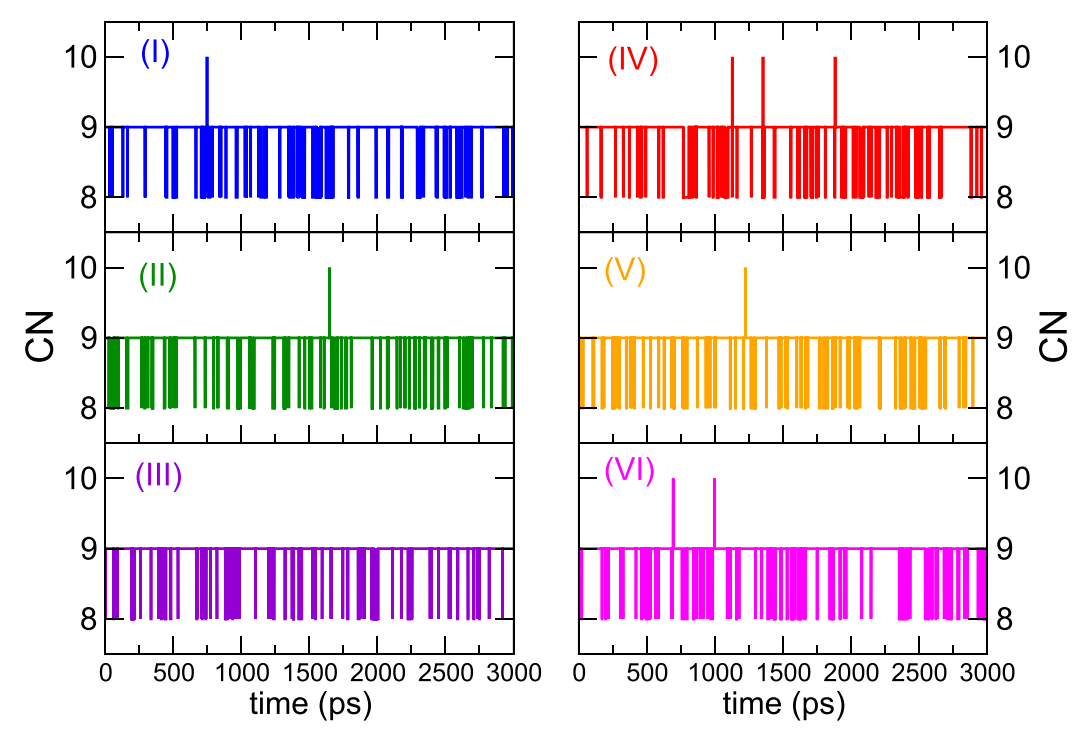

FIG. 9. First shell coordination number as a function of time for six independent molecular dynamics simulations of $\mathrm{Cf}^{3+}$ in aqueous solution as obtained from Pol-MD trajectories.
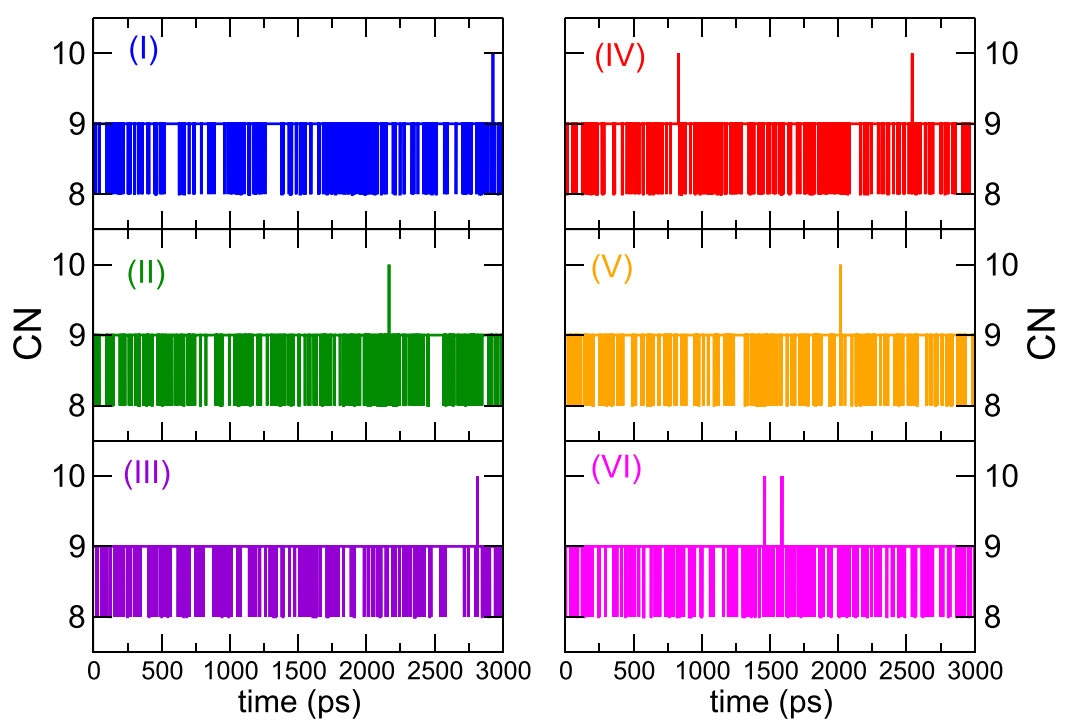

FIG. 10. First shell coordination number as a function of time as obtained from LJ-MD simulations of $\mathrm{Cf}^{3+}$. The $18 \mathrm{~ns}$ trajectory is divided into six to be compared with the equivalent Pol-MD results.

showing small MRTs like $\mathrm{Bk}^{3+}$ (in Pol-MD) and $\mathrm{Cf}^{3+}$ (in both Pol-MD and LJ-MD), the two values are similar showing that the exchange events have time to equilibrate. In the same table (Table IV), we have reported the uncertainties associated with the MRT values, which reflect the lack of convergence for these properties, in particular, in the case of slow exchanges. While for fast exchanges, the uncertainty is about $10 \%$ of the MRT, and it becomes almost comparable with the MRT for slower exchanges.

In the literature, water exchange dynamics data are scarce and experiments were not performed on the $\mathrm{An}^{3+}$ ions. One of the comparable data is the MRT reported by Real et al. on $\mathrm{Cm}^{3+}$ in water, ${ }^{13}$ who provided an average value of $520 \mathrm{ps,}$ which is twice faster than our Pol-MD and ten times faster than LJ-MD. Their results, obtained from different levels of description, report data in the 140-690 ps range, whose upper value is not far from our Pol-LJ value. Other simulations either do not mention the MRT or show too few exchanges to be able to calculate a reliable MRT value.
TABLE IV. Mean residence time, $\tau$, obtained with the direct method over $18 \mathrm{~ns}$ of simulation using polarizable (Pol-MD) and non-polarizable LennardJones (LJ-MD) force fields. Two values of $\mathrm{t}^{*}$ (see definition in Sec. IV B) are reported: $\mathrm{t}^{*}=0.5 \mathrm{ps}$ and $\mathrm{t}^{*}=1.0 \mathrm{ps}$.

\begin{tabular}{lccc}
\hline \hline Ion & Method & $\tau(\mathrm{ps})\left(\mathrm{t}^{*}=0.5 \mathrm{ps}\right)$ & $\tau(\mathrm{ps})\left(\mathrm{t}^{*}=1.0 \mathrm{ps}\right)$ \\
\hline $\mathrm{U}^{3+}$ & Pol-MD & $2745 \pm 1336$ & $3522 \pm 2336$ \\
& LJ-MD & $901 \pm 216$ & $1150 \pm 325$ \\
$\mathrm{~Np}^{3+}$ & Pol-MD & $2793 \pm 1472$ & $2842 \pm 1561$ \\
& LJ-MD & $2945 \pm 1150$ & $3522 \pm 1115$ \\
$\mathrm{Pu}^{3+}$ & Pol-MD & $5586 \pm 3160$ & $7364 \pm 7096$ \\
& LJ-MD & $6231 \pm 2581$ & $6750 \pm 3897$ \\
$\mathrm{Am}^{3+}$ & Pol-MD & $2075 \pm 552$ & $2452 \pm 805$ \\
& LJ-MD & $9000 \pm 3000$ & $10125 \pm 5660$ \\
$\mathrm{Cm}^{3+}$ & Pol-MD & $1064 \pm 191$ & $1274 \pm 286$ \\
& LJ-MD & $5063 \pm 2099$ & $6750 \pm 2756$ \\
$\mathrm{Bk}^{3+}$ & Pol-MD & $606 \pm 155$ & $669 \pm 194$ \\
& LJ-MD & $1541 \pm 590$ & $2129 \pm 1021$ \\
$\mathrm{Cf}^{3+}$ & Pol-MD & $356 \pm 37$ & $398 \pm 40$ \\
& LJ-MD & $333 \pm 26$ & $494 \pm 39$ \\
\hline \hline
\end{tabular}


Note that comparing Pol-MD with LJ-MD results, we do not have a clear tendency in terms of polarization contrary to what observed for lanthanides by first studies of Kowall et al. ${ }^{77}$ in which the unpolarizable force field provides always too fast MRT values with respect to the polarizable one. Note that their polarizable MRT values for lanthanides $\left(\mathrm{Nd}^{3+}, \mathrm{Sm}^{3+}\right.$, and $\mathrm{Yb}^{3+}$ ) are of the same order of magnitude of our results and in agreement with experiments performed on $\mathrm{Ln}^{3+}$ ions in water. ${ }^{78}$ This means that it is possible by using simple LJ potentials to have reasonable MRTs. Unfortunately, there are no experimental data on $\mathrm{An}^{3+}$, and thus it is only possible to note that the differences found in water exchange dynamics between Pol-MD and LJ-MD are not so large and they do not concern all of the elements investigated here.

\section{CONCLUSIONS}

In this work, we have re-parametrized a polarizable force field for studying the actinide(III) ion hydration structure and dynamics based on experimental effective ionic radii recently obtained for these ions in liquid water. ${ }^{27}$ On the same basis, we have derived a non-polarizable LJ based potential. Results are compared with EXAFS experimental data for structural properties providing, for both potentials, an excellent agreement. Furthermore, we have analyzed in detail structural properties obtained with both force fields and water exchange dynamics. Results show that both force fields provide similar properties, with bigger differences in the water exchange dynamics. Experimental data on exchange dynamics are lacking, and thus it is difficult, based on relatively small differences, to determine which force field provides the best results on these properties.

Concluding, this study provides not only a polarizable force field based on ionic properties which can be, in principle, extended to other situations (needing a parametrization of the Buckingham potential like we did, for example, for $\mathrm{Cm}^{3+}$ carbonate $^{79}$ ) but also a simple LJ potential that can be used to study many other systems containing these ions by applying the combination rules to obtain $\mathrm{LJ}$ potentials using the An-An parameters developed here.

\section{ACKNOWLEDGMENTS}

We thank Professor M. A. Denecke and Professor C. Den Auwer for providing us the EXAFS experimental data.

\footnotetext{
${ }^{1}$ N. Kaltsoyannis and P. Scott, "The f elements," in Oxford Chemistry Primers (Oxford University Press, Oxford, 2007).

${ }^{2}$ P. D'Angelo and R. Spezia, Chem. - Eur. J. 18, 11162 (2012).

${ }^{3}$ P. Allen, J. J. Bucher, D. K. Shuh, N. M. Edelstein, and I. Craig, Inorg. Chem. 39, 595 (2000).

${ }^{4}$ B. Brendebach, N. L. Banik, C. M. Marquardt, J. Rothe, M. Denecke, and H. Geckeis, Radiochim. Acta 97, 701 (2009).

${ }^{5} \mathrm{M}$. Antonio, L. Soderholm, C. W. Williams, J.-P. Blaudeau, and B. Bursten, Radiochim. Acta 89, 17 (2001).

${ }^{6} \mathrm{M}$. Antonio, C. Williams, and L. Soderholm, Radiochim. Acta 90, 851 (2002).

${ }^{7}$ R. Revel, C. Auwer, C. Madic, F. David, B. Fourest, S. Hubert, J. Du, and L. R. Morss, Inorg. Chem. 38, 4139 (1999).

${ }^{8}$ S. Skanthakumar, M. Antonio, R. Wilson, and L. Soderholm, Inorg. Chem. 46, 3485 (2007).
}

${ }^{9}$ D. Hagberg, E. Bednarz, N. M. Edelstein, and L. Gagliardi, J. Am. Chem. Soc. 129, 14136 (2007).

${ }^{10}$ E. Galbis, J. Hernandez-Cobos, C. D. Auwer, C. L. Naour, D. Guillaumont, E. Simoni, R. R. Pappalardo, and E. Sanchez-Marcos, Angew. Chem., Int. Ed. 49, 3811 (2010).

${ }^{11}$ E. Galbis, J. Hernandez-Cobos, R. R. Pappalardo, and E. Sanchez-Marcos, J. Chem. Phys. 140, 214104 (2014).

${ }^{12}$ N. Morales, E. Galbis, J. M. Martinez, R. R. Pappalardo, and E. Sanchez-Marcos, J. Phys. Chem. Lett. 7, 4275 (2016).

${ }^{13}$ F. Réal, M. Trumm, B. Schimmelpfennig, M. Masella, and V. Vallet, J. Comput. Chem. 34, 707 (2013).

${ }^{14}$ R. Atta-Fynn, E. J. Bylaska, G. K. Schenter, and W. A. de Jong, J. Phys. Chem. A 115, 4665 (2011).

${ }^{15}$ A. Marjolin, C. Gourlaouen, C. Clavaguera, P. Y. Ren, J.-P. Piquemal, and J.-P. Dognon, J. Mol. Model. 20, 2471 (2014).

${ }^{16}$ S. O. Odoh, E. J. Bylaska, and W. A. de Jong, J. Phys. Chem. A 117, 12256 (2013).

${ }^{17}$ R. Spezia, Y. Jeanvoine, C. Beuchat, L. Gagliardi, and R. Vuilleumier, Phys. Chem. Chem. Phys. 16, 5824 (2014).

${ }^{18}$ P. D'Angelo, A. Zitolo, V. Migliorati, G. Mancini, I. Persson, and G. Chillemi, Inorg. Chem. 48, 10239 (2009).

${ }^{19}$ B. Fourest, J. Duplessis, and F. David, Radiochim. Acta 36, 191 (1984).

${ }^{20}$ A. Villa, B. Hess, and H. Saint-Martin, J. Phys. Chem. B 113, 7270 (2009).

${ }^{21}$ C. Terrier, P. Vitorge, M.-P. Gaigeot, R. Spezia, and R. Vuilleumier, J. Chem. Phys. 133, 044509 (2010).

${ }^{22}$ F. Martelli, R. Vuilleumier, J.-P. Simonin, and R. Spezia, J. Chem. Phys. 137, 164501 (2012).

${ }^{23}$ M. Duvail, R. Spezia, and P. Vitorge, ChemPhysChem 9, 693 (2008).

${ }^{24}$ M. Duvail, P. Vitorge, and R. Spezia, J. Chem. Phys. 130, 104501 (2009).

${ }^{25}$ M. Duvail, F. Martelli, P. Vitorge, and R. Spezia, J. Chem. Phys. 135, 044503 (2011).

${ }^{26}$ R. D. Shannon, Acta Crystallogr., Sect. A 32, 751 (1976).

${ }^{27}$ P. D'Angelo, F. Martelli, R. Spezia, A. Filipponi, and M. A. Denecke, Inorg. Chem. 52, 10318 (2013).

${ }^{28}$ V. Migliorati, A. Serva, F. M. Terenzio, and P. D'Angelo, Inorg. Chem. 56, 6214 (2017).

${ }^{29}$ S. O. Odoh, G. C. Bondarevsky, J. Karpus, Q. Cui, C. He, R. Spezia, and L. Gagliardi, J. Am. Chem. Soc. 136, 17484 (2014).

${ }^{30}$ B. E. Allred, P. B. Rupert, S. S. Gauny, D. D. An, C. Y. Ralston, M. Sturzbecher-Hoehne, R. K. Strong, and R. J. Abergel, Proc. Natl. Acad. Sci. U. S. A. 112, 10342 (2015).

${ }^{31}$ A. Kumar, M. Ali, R. S. Ningthoujam, P. Gaikwad, M. Kumar, B. B. Nath, and B. N. Pandey, J. Hazard. Mater. 307, 281 (2016).

${ }^{32}$ N. Bauer, V. C. Smith, R. T. MacGillivray, and P. J. Panak, Dalton Trans. 44, 1850 (2015).

${ }^{33}$ F. Brulfert, S. Safi, A. Jeanson, E. Martinez-Baez, J. Roques, C. Berthomieu, P. L. Solari, S. Sauge-Merle, and E. Simoni, Inorg. Chem. 55, 2728 (2016).

${ }^{34}$ S. Trumm, P. J. Panak, A. Geist, and T. Fanghanel, Eur. J. Inorg. Chem. 2010, 3022.

${ }^{35}$ S. Tachimori, Y. Sasaki, and S. Suzuki, Solvent Extr. Ion Exch. 20, 687 (2002).

${ }^{36}$ The Chemistry of Actinide and Transactinide Elements, edited by L. R. Morss, N. Edelstein, J. Fuger, and J. J. Katz (Springer, Dordrecht, The Netherlands, 2006).

${ }^{37}$ B. F. Myasoedov, N. P. Molochnikova, V. M. Shkinev, and B. Y. Spivakov, "The behavior of actinides in two-phase aqueous systems based on polyethylene glycol," in Aqueous Biphasic Separations: Biomolecules to Metal Ions, edited by R. D. Rogers and M. A. Eiteman (Springer US, Boston, MA, 1995), pp. 91-99.

${ }^{38}$ K. Binnemans, Chem. Rev. 107, 2592 (2007).

${ }^{39}$ I. Billard, A. Ouadi, and C. Gaillard, Anal. Bioanal. Chem. 400, 1555 (2011).

${ }^{40}$ I. A. Shkrob, T. W. Marin, and M. P. Jensen, Ind. Eng. Chem. Res. 53, 3641 (2014).

${ }^{41}$ M. Duvail, M. Souaille, R. Spezia, T. Cartailler, and P. Vitorge, J. Chem. Phys. 127, 034503 (2007).

${ }^{42}$ P. D'Angelo, A. Zitolo, V. Migliorati, G. Chillemi, M. Duvail, P. Vitorge, S. Abadie, and R. Spezia, Inorg. Chem. 50, 4572 (2011).

${ }^{43}$ B. T. Thole, Chem. Phys. 59, 341 (1981).

${ }^{44}$ M. Souaille, H. Loirat, D. Borgis, and M.-P. Gaigeot, Comput. Phys. Commun. 180, 276 (2009).

${ }^{45}$ M. Sprik, J. Chem. Phys. 95, 2283 (1991).

${ }^{46}$ W. L. Jorgensen, J. Chandrasekhar, J. D. Madura, R. W. Impey, and M. L. Klein, J. Chem. Phys. 79, 926 (1983). 
${ }^{47}$ P. van Duijnen and M. Swart, J. Phys. Chem. A 102, 2399 (1998).

${ }^{48}$ F. Martelli, S. Abadie, J.-P. Simonin, R. Vuilleumier, and R. Spezia, Pure Appl. Chem. 85, 237 (2013).

${ }^{49}$ V. Migliorati and P. D’Angelo, Inorg. Chem. 55, 6703 (2016).

${ }^{50}$ P. D'Angelo, V. Migliorati, F. Sessa, G. Mancini, and I. Persson, J. Phys. Chem. B 120, 4114 (2016).

${ }^{51}$ V. Migliorati, G. Mancini, S. Tatoli, A. Zitolo, A. Filipponi, S. De Panfilis, A. Di Cicco, and P. D'Angelo, Inorg. Chem. 52, 1141 (2013).

${ }^{52}$ V. Migliorati, A. Zitolo, G. Chillemi, and P. D'Angelo, ChemPlusChem 77, 234 (2012)

${ }^{53}$ V. Migliorati, G. Mancini, G. Chillemi, A. Zitolo, and P. D'Angelo, J. Phys. Chem. A 115, 4798 (2011).

${ }^{54}$ H. J. C. Berendsen, J. R. Grigera, and T. P. Straatsma, J. Phys. Chem. 91, 6269 (1987).

${ }^{55}$ M. Duvail, R. Spezia, T. Cartailler, and P. Vitorge, Chem. Phys. Lett. 448, 41 (2007).

${ }^{56}$ M. Duvail, P. Vitorge, and R. Spezia, Chem. Phys. Lett. 498, 90 (2010).

${ }^{57}$ U. Essmann, L. Perera, M. L. Berkowitz, T. Darden, H. Lee, and L. G. Pedersen, J. Chem. Phys. 103, 8577 (1995).

${ }^{58}$ H. J. C. Berendsen, D. van der Spoel, and R. van Drunen, Comput. Phys. Commun. 91, 43 (1995).

${ }^{59}$ S. Nosé, J. Chem. Phys. 81, 511 (1984).

${ }^{60}$ D. J. Evans and B. L. Holian, J. Chem. Phys. 83, 4069 (1985).

${ }^{61}$ R. W. Impey, P. A. Madden, and I. R. McDonald, J. Phys. Chem. 87, 5071 (1983).

${ }^{62}$ T. S. Hofer, H. T. Tran, C. F. Schwenk, and B. M. Rode, J. Comput. Chem. 25, 211 (2004).

${ }^{63}$ A. Filipponi and A. Di Cicco, Phys. Rev. B 52, 15135 (1995).
${ }^{64}$ A. Filipponi, A. Di Cicco, and C. R. Natoli, Phys. Rev. B 52, 15122 (1995).

${ }^{65}$ E. Burattini, P. D'Angelo, E. Giglio, and N. V. Pavel, J. Phys. Chem. 95, 7880 (1991).

${ }^{66}$ P. D'Angelo, A. Lapi, V. Migliorati, A. Arcovito, M. Benfatto, O. M. Roscioni, W. Meyer-Klaucke, and S. Della-Longa, Inorg. Chem. 47, 9905 (2008).

${ }^{67}$ P. D'Angelo, N. V. Pavel, D. Roccatano, and H.-F. Nolting, Phys. Rev. B 54, 12129 (1996).

${ }^{68}$ V. Pham, I. Tavernelli, C. Milne, R. van der Veen, P. D’Angelo, C. Bressler, and M. Chergui, Chem. Phys. 371, 24 (2010).

${ }^{69}$ P. D'Angelo, A. Di Nola, A. Filipponi, N. V. Pavel, and D. Roccatano, J. Chem. Phys. 100, 985 (1994).

${ }^{70}$ P. D'Angelo, V. Migliorati, G. Mancini, V. Barone, and G. Chillemi, J. Chem. Phys. 128, 084502 (2008).

${ }^{71}$ P. D’Angelo, G. Chillemi, V. Barone, G. Mancini, N. Sanna, and I. Persson, J. Phys. Chem. B 109, 9178 (2005).

${ }^{72}$ P. Allen, J. J. Bucher, D. K. Shuh, N. M. Edelstein, and T. Reich, Inorg. Chem. 36, 4676 (1997).

${ }^{73}$ P. D'Angelo, S. De Panfilis, A. Filipponi, and I. Persson, Chem. - Eur. J. 14, 3045 (2008).

${ }^{74}$ P. D'Angelo, V. Migliorati, and L. Guidoni, Inorg. Chem. 49, 4224 (2010).

${ }^{75}$ V. Migliorati, F. Sessa, G. Aquilanti, and P. D'Angelo, J. Chem. Phys. 141, 044509 (2014).

${ }^{76}$ V. Migliorati and P. D’Angelo, RSC Adv. 3, 21118 (2013).

${ }^{77}$ T. Kowall, F. Foglia, L. Helm, and A. E. Merbach, J. Am. Chem. Soc. 117, 3790 (1995).

${ }^{78}$ L. Helm and A. E. Merbach, Chem. Rev. 105, 1923 (2005).

${ }^{79}$ R. Spezia, Y. Jeanvoine, and R. Vuilleumier, J. Mol. Model. 20, 2398 (2014). 